\title{
Searching for muon to electron conversion: The Mu2e experiment at Fermilab
}

\author{
Richard Bonventre $^{1 \star}$ on behalf of the Mu2e Collaboration
}

1 Lawrence Berkeley National Lab, Berkeley, USA

$\star$ rbonventre@lbl.gov

Proceedings for the 15th International Workshop on Tau Lepton Physics, Amsterdam, The Netherlands, 24-28 September 2018

doi:10.21468/SciPostPhysProc. 1

\begin{abstract}
The Mu2e experiment will measure the charged-lepton flavor violating (CLFV) neutrinoless conversion of a negative muon into an electron in the field of a nucleus. Mu2e will improve the previous measurement by four orders of magnitude, reaching a $\mathbf{9 0 \%}$ C.L. limit of $8 \times 10^{-17}$ on the conversion rate. The experiment will reach mass scales of nearly $10^{4} \mathrm{TeV}$, far beyond the direct reach of colliders. The experiment is sensitive to a wide range of new physics, complementing and extending other CLFV searches. Mu2e is under design and construction at the Muon Campus of Fermilab; we expect to start taking physics data in 2022 with 3 years of running to achieve our target sensitivity.
\end{abstract}

(c) (1) Copyright R. Bonventre et al. This work is licensed under the Creative Commons Attribution 4.0 International License. Published by the SciPost Foundation.
Received 06-11-2018

Accepted 15-01-2019

Published 21-02-2019

doi:10.21468/SciPostPhysProc. 1.038

\section{Introduction}

Measurements of neutrino oscillations show that lepton flavor conservation is strongly violated in the neutrino sector. In the Standard Model this violation can be explained through the addition of nonzero neutrino masses. The scale of these masses, however, strongly suppresses Standard Model contributions to charged lepton flavor violating (CLFV) processes. For example, the branching fraction for $\mu \rightarrow e \gamma$ decays is predicted to be less than $10^{-54}$. Thus any detection of CLFV experimentally is a definitive sign of new physics. Many theories of physics beyond the Standard Model enhance CLFV processes due to the addition of new particles and interactions, and this enhancement can raise the rate to a detectable level [1-3].

There are several CLFV processes that can be studied, each with differing sensitivities to various models of new physics. In general, although $\tau$ decays have the advantage of a much larger phase space, muon decay channels are the most sensitive due to the ability to create intense muon beams. The current most restrictive limits on CLFV in muon processes come from MEG, with a limit of $4.2 \times 10^{-13}$ on the branching fraction of $\mu \rightarrow e \gamma$ [4], and SINDRUM-II with a limit of $\mathrm{R}_{\mu e}<6 \times 10^{-13}$ for muon conversion with a gold target [5]. 
We can compare the sensitivity of the different processes to new physics by considering generic contributions to CLFV with an effective Lagrangian given by

$$
\mathcal{L}_{\mathrm{CLFV}}=\frac{m_{\mu}}{(1+\kappa) \Lambda^{2}} \bar{\mu}_{R} \sigma_{\mu \nu} e_{L} F^{\mu \nu}+\frac{\kappa}{(1+\kappa) \Lambda^{2}} \bar{\mu}_{L} \gamma_{\mu} e_{L}\left(\sum_{q=u, d} \overline{q_{L}} \gamma^{\mu} q_{L}\right),
$$

with the parameter $\kappa$ determining the relative strength of "loop" terms and "contact terms" and $\lambda$ denoting the mass scale of the physics (assuming unit coupling) [6]. Both $\mu \rightarrow e \gamma$ and $\mu N \rightarrow e N$ measurements are sensitive to the new physics if loop terms dominate $(\kappa \ll 1)$, but only $\mu N \rightarrow e N$ is sensitive if contact terms do $(\kappa \gg 1)$. The sensitivity of Mu2e will allow it to probe effective mass scales up to $10^{4} \mathrm{TeV}$, beyond the range of collider experiments.

\section{The Mu2e experiment}

The Mu2e experiment will search for neutrino-less conversion of a negative muon into an electron in the field of a nucleus [7]. Slow muons are stopped on an aluminum target producing muonic atoms, which quickly drop to the lowest energy 1s state. Once in this state the muons decay, convert, or are captured by the nucleus, with a livetime of 864 ns. For negative muons, decays or conversions of the bound muon happen coherently with the nucleus. When the muon undergoes CLFV conversion, the outgoing electron effectively takes all the rest-mass energy of the muon, minus the atomic binding energy. The signal produced by this process is thus a mono-energetic electron with an energy of approximately $105 \mathrm{MeV}$. The parameter measured by Mu2e and other muon conversion experiments is the rate of this CLFV conversion process normalized to the rate of muon capture by the nucleus:

$$
R_{\mu e}=\frac{\mu^{-}+A(Z, N) \rightarrow e^{-}+A(Z, N)}{\mu^{-}+A(Z, N) \rightarrow v_{\mu}+A(Z-1, N)} .
$$

There are three primary sources of backgrounds for this signal: muons decaying in orbit, cosmic rays, and the muon beam itself. When muons stopped on aluminum undergo decayin-orbit (DIO), the coherent recoil of the nucleus also modifies the Michel energy spectrum endpoint, pushing it all the way up to the conversion peak energy, as shown in Fig. 1. Near the conversion endpoint the DIO energy spectrum falls sharply as $\left(E-E_{\text {endpoint }}\right)^{5}$. The electrons produced by DIO are thus a background to the conversion search that must be separated using a precise momentum measurement. Cosmic rays can scatter off the aluminum stopping target and produce $105 \mathrm{MeV}$ electrons that look identical to the signal. Since these background events cannot be separated from the signal by any other means, an active cosmic ray veto is needed to reject them. Finally, the creation of the muon beam produces many possible sources of background. For example, pions that reach the stopping target can undergo radiative pion capture (RPC), producing a gamma that can then convert, producing a conversion electron-like signal in the detector.

The RPC background limited the sensitivity of previous muon conversion experiments. Mu2e will use a pulsed muon beam, allowing it to use timing cuts to reject these events. An $8 \mathrm{GeV} 8 \mathrm{~kW}$ pulsed proton beam from the Fermilab booster is used to create the muons, with beam bunches separated by $1.7 \mu \mathrm{s}$. The backgrounds associated with the beam, like RPC events, are prompt in time, while the signal events are fairly uniform due to the 864 ns livetime of stopped muons on aluminum. Thus, by delaying the signal window, the beam background events can be reduced dramatically, as shown in Fig. 2. In order to avoid any late pions arriving inside the event window, Mu2e requires a beam extinction, or ratio of in-time to out-of-time protons, of less than $10^{-10}$. Mu2e will stop approximately $10^{18}$ muons in total in order to reach its target sensitivity. 


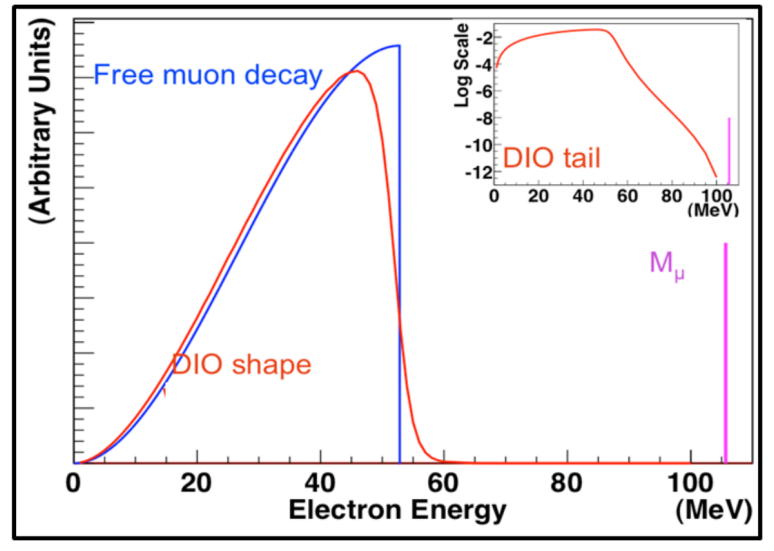

Figure 1: Energy spectrum of electrons produced by muon decay-in-orbit (red) compared with those from free muon decay (blue). Near the conversion endpoint at 105 $\mathrm{MeV}$ the decay-in-orbit spectrum falls as $\left(E-E_{\text {endpoint }}\right)^{5}$.

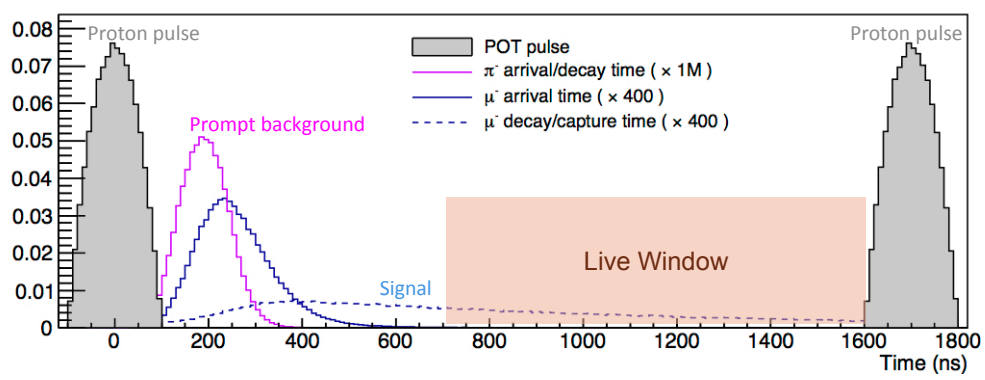

Figure 2: The Mu2e muon beam structure and live window compared to expected time distributions of backgrounds and signals.

\section{Experimental design}

The layout of the Mu2e experiment is shown in Fig. 3. It consists of three regions of superconducting solenoids: the production solenoid (PS), transport solenoid (TS), and detector solenoid (DS). The proton beam enters the PS and hits a tungsten production target. The PS produces a graded field that reflects low momentum pions and muons back towards the TS, which greatly increases collection efficiency and the intensity of the final muon beam. The TS has two curved sections which separate particles in the beam by charge and momentum, and allow for the selection of low momentum muons. The DS contains the aluminum stopping target, which consists of a series of thin foils, as well as a straw tracker and an electromagnetic calorimeter. The stopping target again sits in a gradient field, reflecting conversion electrons towards the detector elements. The straw tracker and electromagnetic calorimeter sit in a constant $1 \mathrm{~T}$ field. The tracker is designed to provide the high precision momentum measurement that allows for the rejection of the DIO background events, while the calorimeter provides a separate energy and time measurement that provide particle identification and a seed for the reconstruction.

The tracker is made of approximately 20,000 low mass straws in vacuum, each $5 \mathrm{~mm}$ in diameter and made of $15 \mu \mathrm{m}$ thick mylar. The straws are oriented transverse to the solenoid axis, arranged in 120 degree arc "panels". Each panel contains 96 straws in two staggered layers. Twelve panels oriented by successive 30 degree rotations make up a station, providing stereo position information. The tracker consists of 18 stations in total, giving it a total length 


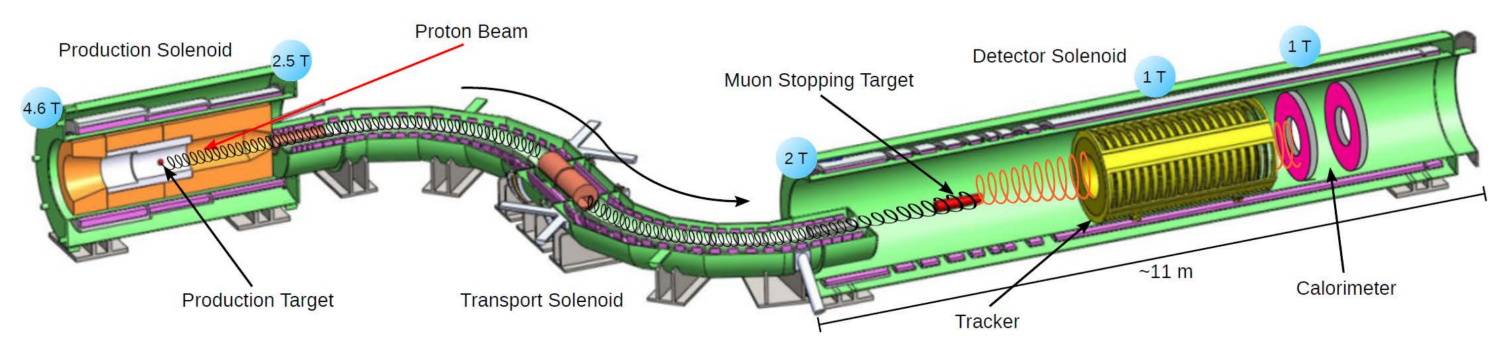

Figure 3: Layout of the Mu2e experiment.

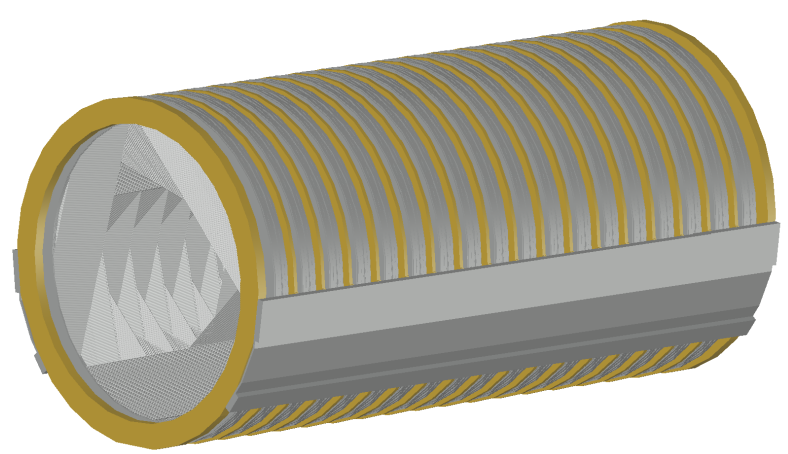

Figure 4: Sketch of the Mu2e straw tracker. Straws are arranged into 120 degree arcs oriented transverse to the solenoid axis, with 12 panels making up a station. The whole tracker consists of 18 stations and is 3.2 meters long.

of $3.2 \mathrm{~m}$, as shown in Fig. 4. The straws do not reach within a minimum radius of $38 \mathrm{~cm}$, making the tracker blind to the majority of the beam flash and low momentum DIO electrons. Signals from the straws are digitized at both ends, allowing for the determination of hit positions both transverse to and longitudinally along the wire. A digital waveform is also recorded in order to allow the rejection of highly ionizing hits from protons.

An eight channel tracker prototype, shown in Fig. 5, has been used to measure the performance of the tracker design using cosmic rays. Fig. 6 shows the transverse resolution, longitudinal resolution, and the efficiency of the cosmic ray data compared to predictions from simulations. The transverse resolution is found to have a full width half max of $283 \mu \mathrm{m}$, while the longitudinal resolution is found to have a core resolution of $43 \pm 1 \mathrm{~mm}$, meeting the requirements of the tracker. In addition to the eight channel prototype, full size pre-production panels have been assembled and tested, and multiple panels have been assembled into a prototype station. Final straw production is currently underway.

The electromagnetic calorimeter is located downstream of the tracker, and provides an independent energy and time measurement that is necessary for particle identification [8]. It consists of two annular disks, each containing 674 undoped CsI scintillating crystals of dimension $3.4 \times 3.4 \times 20 \mathrm{~cm}^{3}$ read out by custom silicon photomultipliers (SiPMs), as shown in Fig. 7. The disks are separated by a distance that maximize the chance of at least one being hit by any conversion electron. In order to provide e/ $\mu$ separation, the calorimeter is required to have an energy resolution of approximately $5 \%$ and a time resolution of better than 500 ps. A 3 by 3 CsI crystal array prototype was tested at the Frascati Beam Test Facility with electron beams of 80 to $120 \mathrm{MeV}$, and an energy resolution of $6.5 \%$ and time resolution of 100 ps for $100 \mathrm{MeV}$ particles was demonstrated, as shown in Fig. 8 [9]. A larger scale prototype consisting of 51 crystals and 102 SiPMs has also been built testing integration and assembly 


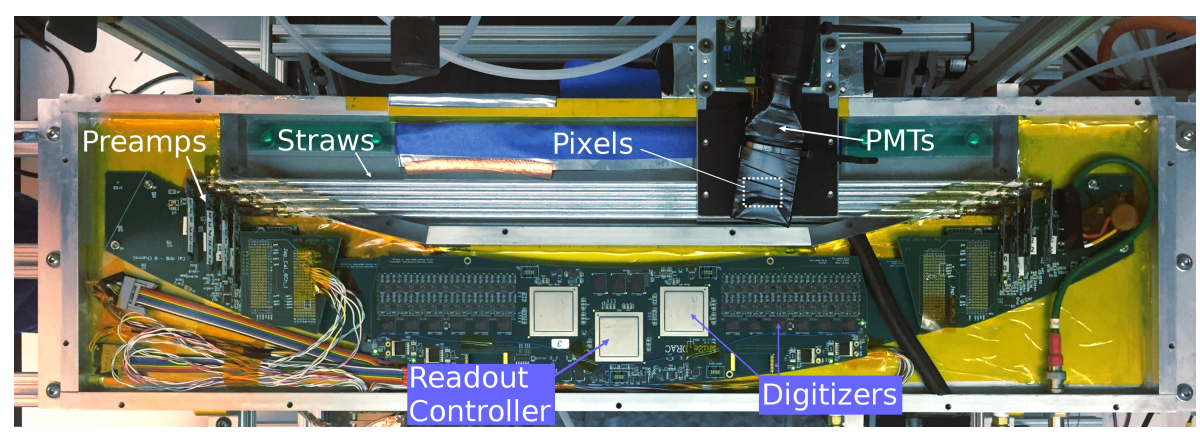

Figure 5: Eight straw tracker prototype used to measure hit resolution and efficiency with cosmic rays. Prototype tracker electronics, including preamplifiers on each straw end and FPGA based digitizers and readout controllers, are used to measure the straw signals. A cosmic ray trigger was built using two PMTs and a custom triggering board. Trajectories of the cosmic rays was determined using FEI4 ATLAS pixel detectors.
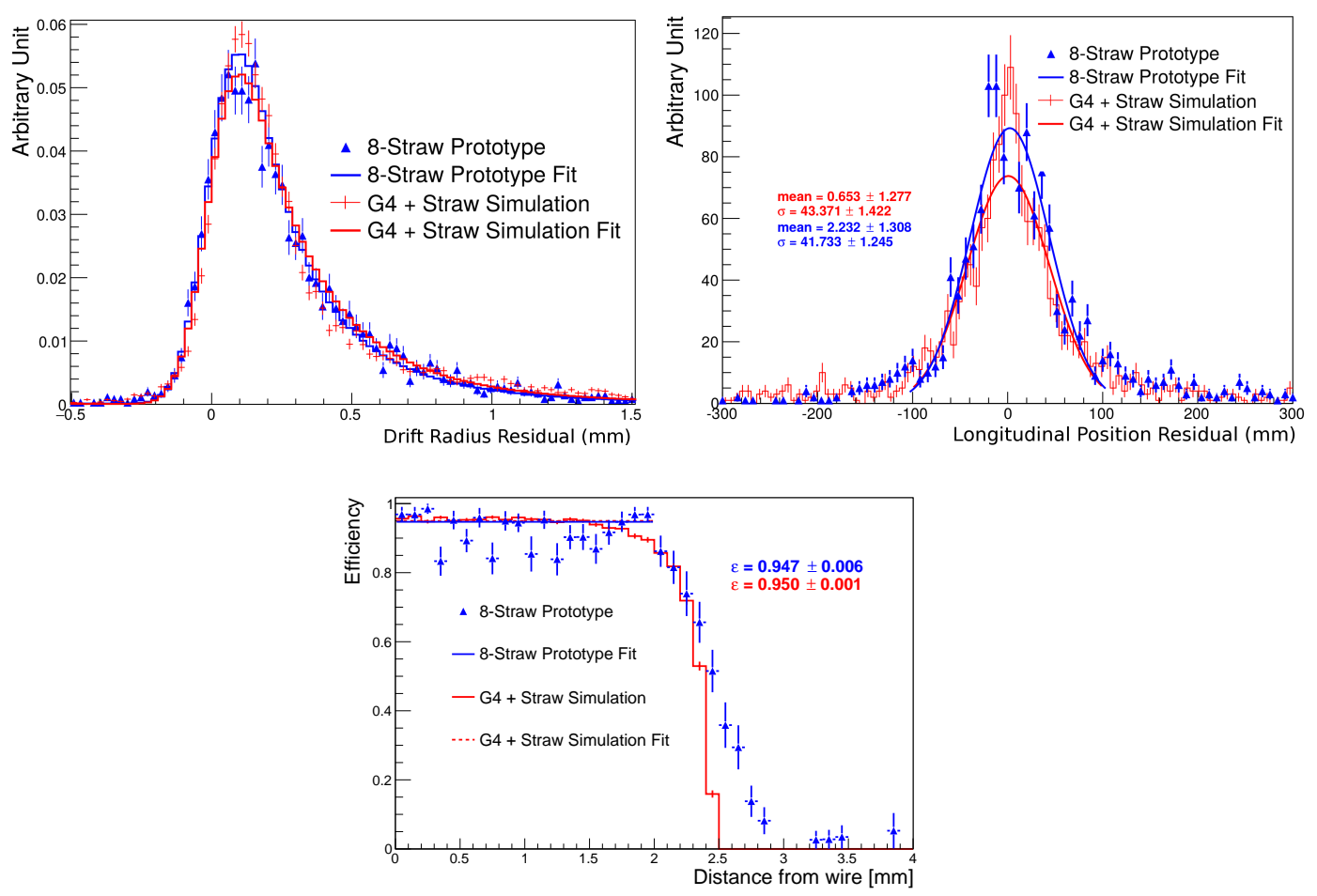

Figure 6: Transverse resolution (top-left), longitudinal resolution (top-right), and efficiency (bottom) for cosmic ray events from an eight channel tracker prototype. Data is shown in blue, while results of Monte Carlo simulation of the prototype setup are shown in red. The transverse residuals are fit to a function that includes both a Gaussian smearing component as well as a exponential component that includes geometric effects. The longitudinal resolution is determined using a Gaussian fit to the core of the residual distribution. The efficiency quoted is determined by a fit to events less than $2 \mathrm{~mm}$ from the wire. 


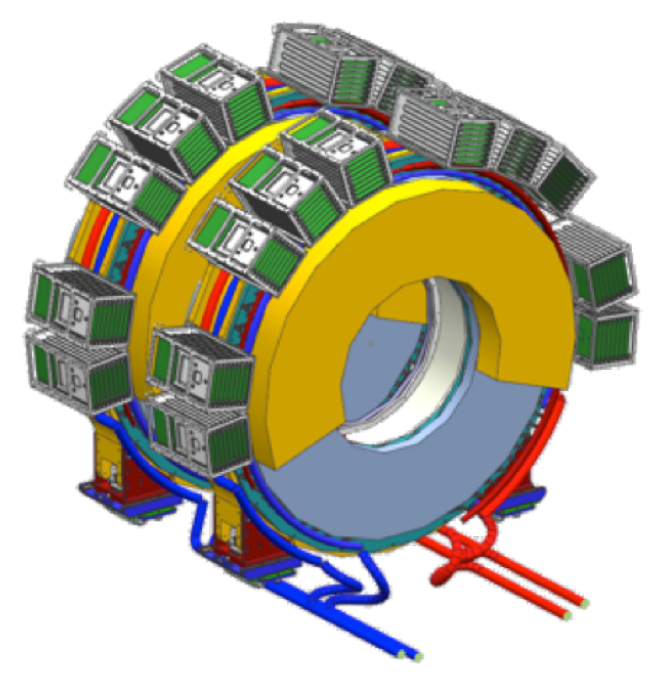

Figure 7: CAD drawing of the electromagnetic calorimeter. The calorimeter consists of two annular disks, with the crystals in each located between an inner and outer aluminum ring. In the figure, the inner ring is visible in gray at the center of the closer disk. The digitizing electronics are in the crates on the outside of the disks. The light blue area represents the support for the SiPMs and front end electronics, and the yellow area represents the envelope of the cables from the FEE to the digitizing electronics.

procedures, shown in Fig. 9 [10].

In order to reject events produced by cosmic rays, the entire DS and half of the TS are covered by an active cosmic ray veto (CRV) detector, as shown in Fig. 10. In order to reach the Mu2e sensitivity goals the CRV must have a veto efficiency of $99.99 \%$ for a reasonable deadtime contribution while running in a high radiation environment. The CRV is made up of four overlapping layers of scintillating bars, each with two embedded wavelength shifting fibers connected to Hamamatsu SiPMs. A three out of four coincidence is required to activate the veto. Test beam measurements have been performed on a full size prototype bar, and the light yield was found to meet specifications [11].
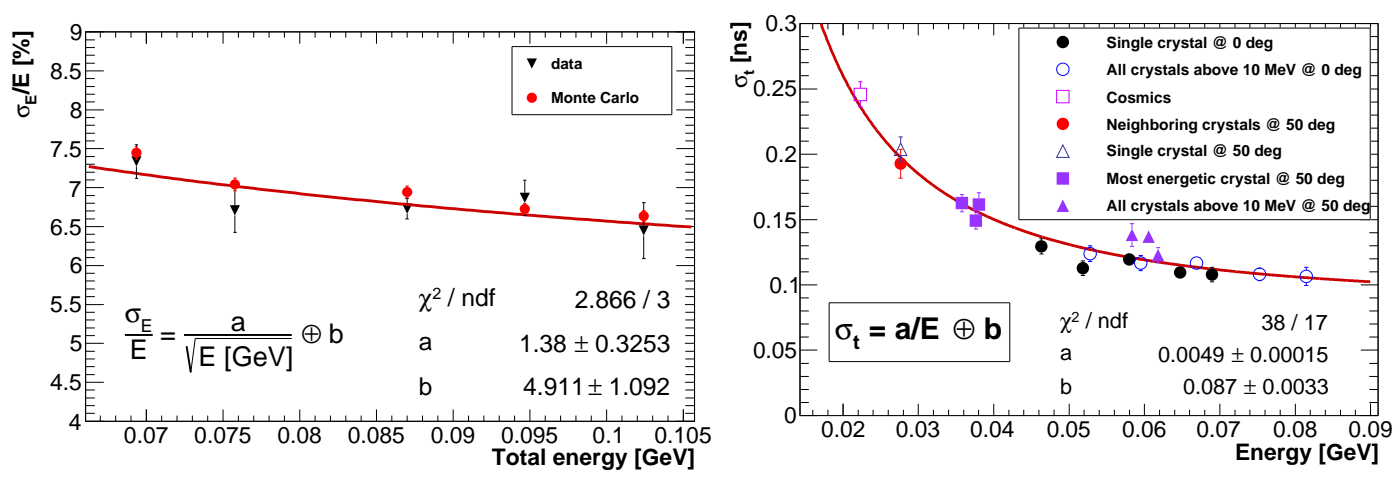

Figure 8: Energy resolution (left) and time resolution (right) obtained from a 3 by 3 CsI crystal array prototype using an electron beam from the Frascati Beam Test Facility. 


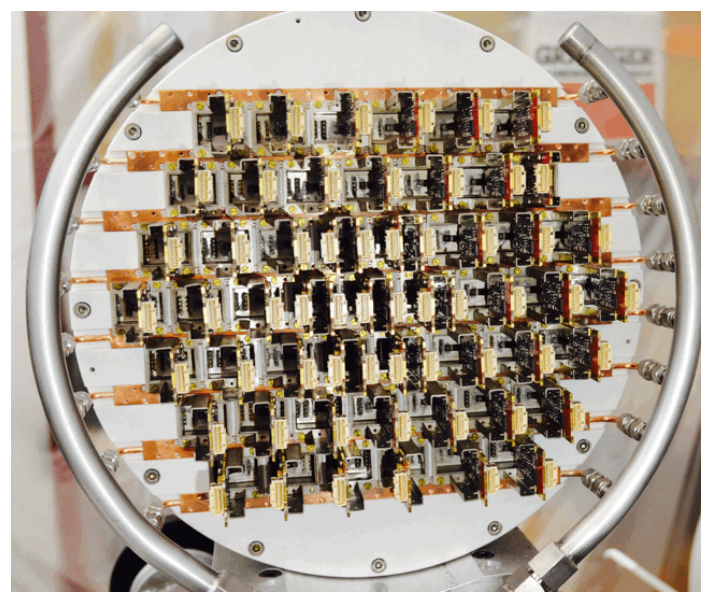

Figure 9: Larger calorimeter prototype consisting of 51 crystals and 102 SiPMs.
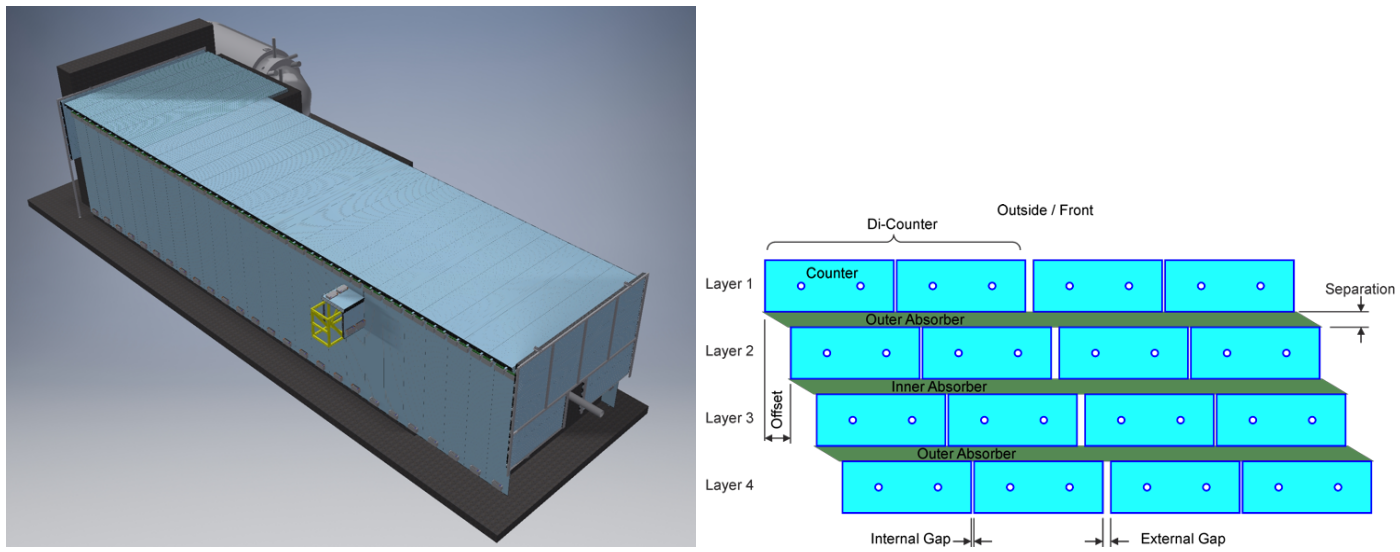

Figure 10: Layout of the cosmic ray veto (left), and cross sectional view (right).

\section{Simulation and sensitivity prediction}

A Geant-4 based simulation of the full experiment from the production target forward is used to predict the expected signals, backgrounds, and conversion sensitivity. The simulation includes a detailed model of the straw tracker response tuned to low level measurements of gains, thresholds, and pulse shapes from the eight channel prototype and validated with the resolution and efficiency measurements shown in Fig. 6. A Kalman Filter algorithm is used to reconstruct particle tracks and momentum. Fig. 11 shows the simulated reconstructed momentum spectra for backgrounds and signals near the conversion endpoint. It assumes a total of $3 \times 10^{20}$ protons on target, and to normalize the signal assumes $R_{\mu e}=2 \times 10^{-16}$. A signal window in momentum is selected around the conversion endpoint to maximize discovery sensitivity. The backgrounds included in this signal window are given in Table 1, with a total expectation of $0.41 \pm 0.13$ events after the full run. With this signal window and background, Mu2e will have a $5 \sigma$ discovery reach of $R_{\mu e} \geq 2 \times 10^{-16}$, or be able to set a limit at a $90 \%$ C.L. of $R_{\mu e} \leq 8 \times 10^{-17}$. 


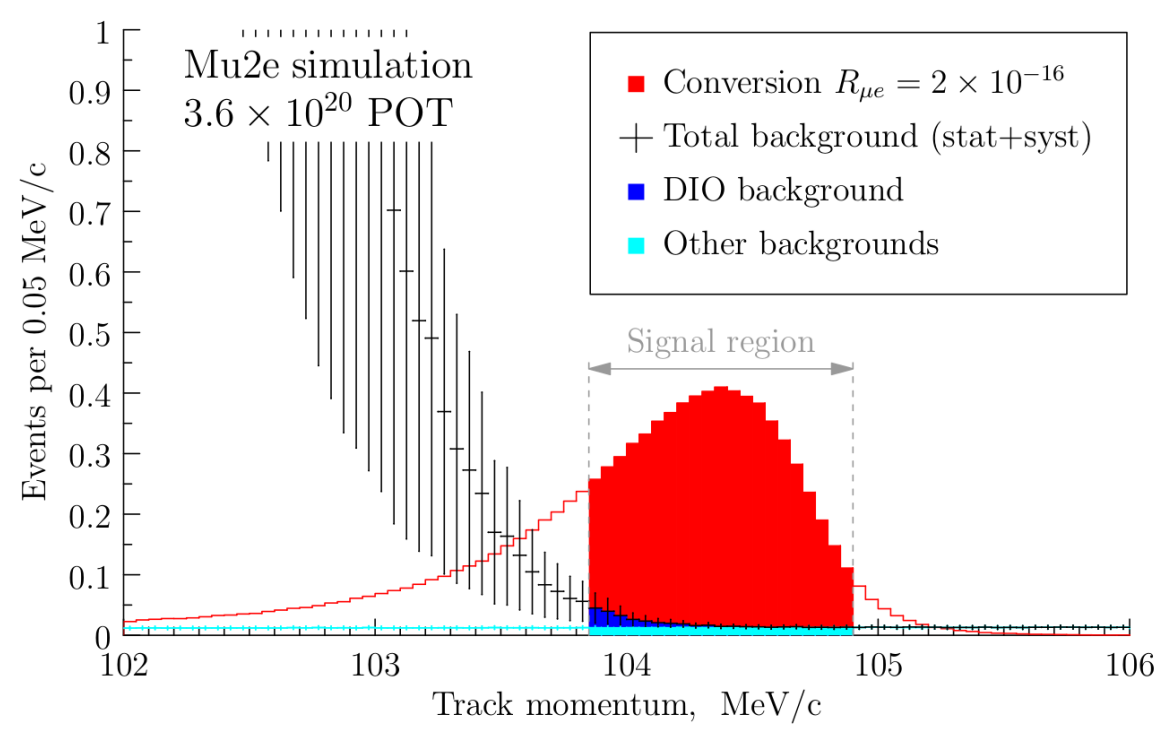

Figure 11: Monte Carlo simulation prediction of reconstructed momentum spectra for background and signal events.

Table 1: Expected backgrounds in signal window for $3 \times 10^{20}$ protons on target in a 3 year run.

\begin{tabular}{cc}
\hline \hline Process & Expected event yield \\
\hline Cosmic ray muons & $0.21 \pm 0.02$ (stat) \pm 0.06 (syst) \\
DIO & $0.14 \pm 0.03$ (stat) \pm 0.11 (syst) \\
Antiprotons & $0.040 \pm 0.001$ (stat) \pm 0.020 (syst) \\
Pion capture & $0.021 \pm 0.001$ (stat) \pm 0.002 (syst) \\
Muon decay-in-flight & $<0.003$ \\
Pion decay-in-flight & $0.001 \pm 0.001$ \\
Beam electrons & $(2.1 \pm 1.0) \times 10^{-4}$ \\
Radiative muon capture & $0.000_{-0.000}^{+0.004}$ \\
\hline Total & $0.41 \pm 0.13$ (stat + syst $)$ \\
\hline \hline
\end{tabular}

\section{Status and Summary}

The Mu2e experiment at Fermilab will search for CLFV muon conversion with a sensitivity four orders of magnitude improved over current limits. An intense pulsed proton beam, large graded solenoids, a high precision straw tracker and electromagnetic calorimeter, and an efficiency cosmic ray veto will allow it to reach a $90 \%$ confidence limit of $R_{\mu e} \leq 8 \times 10^{-17}$ after 3 years of running. Prototypes of the detector elements have been produced and used to show that the designs meet specifications. Construction of all three superconducting solenoids has begun. 95\% of the TS coils have been wound, and a TS test module has been delivered and tested at Fermilab, and found to pass all specifications. Physics data taking is expected to begin in 2022. 


\section{Acknowledgements}

We are grateful for the vital contributions of the Fermilab staff and the technical staff of the participating institutions.

Funding information This work was supported by the US Department of Energy; the Italian Istituto Nazionale di Fisica Nucleare; the Science and Technology Facilities Council, UK; the Ministry of Education and Science of the Russian Federation; the US National Science Foundation; the Thousand Talents Plan of China; the Helmholtz Association of Germany; and the EU Horizon 2020 Research and Innovation Program under the Marie Sklodowska-Curie Grant Agreement No.690835. This document was prepared by members of the Mu2e Collaboration using the resources of the Fermi National Accelerator Laboratory (Fermilab), a U.S. Department of Energy, Office of Science, HEP User Facility. Fermilab is managed by Fermi Research Alliance, LLC (FRA), acting under Contract No. DE-AC02-07CH11359.

\section{References}

[1] Y. Kuno and Y. Okada, Muon decay and physics beyond the standard model, Rev. Mod. Phys. 73, 151 (2001), doi:10.1103/RevModPhys.73.151.

[2] W. J. Marciano, T. Mori and J. M. Roney, Charged lepton flavor violation experiments, Annu. Rev. Nucl. Part. Sci. 58, 315 (2008), doi:10.1146/annurev.nucl.58.110707.171126.

[3] M. Raidal et al., Flavor physics of leptons and dipole moments, Eur. Phys. J. C 57, 13 (2008), doi:10.1140/epjc/s10052-008-0715-2.

[4] A. M. Baldini et al., Search for the lepton flavour violating decay $\mu^{+} \rightarrow \mathrm{e}^{+} \gamma$ with the full dataset of the MEG experiment, Eur. Phys. J. C 76, 434 (2016), doi:10.1140/epjc/s10052016-4271-x.

[5] W. Bertl et al., A search for $\mu$-e conversion in muonic gold, Eur. Phys. J. C 47, 337 (2006), doi:10.1140/epjc/s2006-02582-x.

[6] A. de Gouvêa, (Charged) lepton flavor violation, Nucl. Phys. B - Proc. Suppl. 188, 303 (2009), doi:10.1016/j.nuclphysbps.2009.02.071.

[7] L. Bartoszek et al., Mu2e technical design report (2015), arXiv:1501.05241.

[8] N. Atanov et al., Design and status of the Mu2e electromagnetic calorimeter, Nucl. Instrum. Methods Phys. Res. Sect. A 824, 695 (2016), doi:10.1016/j.nima.2015.09.074.

[9] O. Atanova et al., Measurement of the energy and time resolution of a undoped CsI + MPPC array for the Mu2e experiment, J. Inst. 12, P05007 (2017), doi:10.1088/17480221/12/05/P05007.

[10] N. Atanov et al., Design and status of the Mu2e crystal calorimeter, IEEE Trans. Nucl. Sci. 65, 2073 (2018), doi:10.1109/TNS.2018.2790702.

[11] A. Artikov et al., Photoelectron yields of scintillation counters with embedded wavelengthshifting fibers read out with silicon photomultipliers, Nucl. Instrum. Methods Phys. Res. Sect. A 890, 84 (2018), doi:10.1016/j.nima.2018.02.023. 